\title{
Conformable Fractional Integrals Versions of Hermite-Hadamard Inequalities and Their Generalizations
}

\author{
Muhammad Adil Khan, ${ }^{1,2}$ Yu-Ming Chu $\left(\mathrm{D},{ }^{3}\right.$ Artion Kashuri, ${ }^{4}$ \\ Rozana Liko, ${ }^{4}$ and Gohar Ali $\left(\mathbb{D}^{5}\right.$ \\ ${ }^{1}$ College of Science, Hunan City University, Yiyang 413000, China \\ ${ }^{2}$ Department of Mathematics, University of Peshawar, Peshawar 25000, Pakistan \\ ${ }^{3}$ Department of Mathematics, Huzhou University, Huzhou 3132000, China \\ ${ }^{4}$ Department of Mathematics, Faculty of Technical Science, University "Ismail Qemali", Vlorë, Albania \\ ${ }^{5}$ Department of Mathematics, Islamia College, Peshawar 25000, Pakistan
}

Correspondence should be addressed to Yu-Ming Chu; chuyuming@zjhu.edu.cn

Received 5 September 2017; Accepted 21 December 2017; Published 29 May 2018

Academic Editor: Maria Alessandra Ragusa

Copyright (C) 2018 Muhammad Adil Khan et al. This is an open access article distributed under the Creative Commons Attribution License, which permits unrestricted use, distribution, and reproduction in any medium, provided the original work is properly cited.

We prove new Hermite-Hadamard inequalities for conformable fractional integrals by using convex function, $s$-convex, and coordinate convex functions. We prove new Montgomery identity and by using this identity we obtain generalized HermiteHadamard type inequalities.

\section{Introduction}

The class of convex functions is well known in the literature and is usually defined in the following way: let I be an interval in $\mathbb{R}$; then a function $f: I \rightarrow \mathbb{R}$ is said to be convex on $I$ if the inequality

$$
f(\lambda x+(1-\lambda) y) \leq \lambda f(x)+(1-\lambda) f(y)
$$

holds for all $x, y \in I$ and $\lambda \in[0,1]$. Also, we say that $f$ is concave, if the inequality in (1) holds in the reverse direction. There are several generalizations of the convex function. Here we mention basic definition of $s$-convex function and coordinate convex function. In the paper [1], Hudzik and Maligranda considered a generalization of convex function, which is known as s-convex function in the second sense. This class of function is defined in the following way: a function $f:[0,+\infty) \rightarrow \mathbb{R}$ is said to be $s$-convex in the second sense if

$$
f(\lambda x+(1-\lambda) y) \leq \lambda^{s} f(x)+(1-\lambda)^{s} f(y)
$$

holds for all $x, y \geq 0$ and $\lambda \in[0,1]$ and for some fixed $s \in$ $(0,1]$. The class of $s$-convex functions in the second sense is usually denoted by $K_{s}^{2}$.
In [2], the concept of convex functions defined on the coordinates of the bidimensional interval of the plane of two variables was introduced.

Definition 1. Let us consider the bidimensional interval $\Delta:=$ $[a, b] \times[c, d]$ in $\mathbb{R}^{2}$ with $a<b$ and $c<d$. A function $\phi$ : $[a, b] \times[c, d] \rightarrow \mathbb{R}$ is called convex on the coordinates if the partial mappings $\phi_{y}:[a, b] \rightarrow \mathbb{R}$ defined as $\phi_{y}(t):=\phi(t, y)$ and $\phi_{x}:[c, d] \rightarrow \mathbb{R}$ defined as $\phi_{x}(s):=\phi(x, s)$ are convex for all $x \in[a, b]$ and $y \in[c, d]$.

Remark 2. Note that every convex function $\phi:[a, b] \times$ $[c, d] \rightarrow \mathbb{R}$ is convex on the coordinates, but the converse is not generally true [2].

Many important inequalities have been obtained for this class of functions but here we will present only one of them.

If $f: I \rightarrow \mathbb{R}$ is a convex function on the interval $\mathrm{I}$, then, for any $a, b \in \mathrm{I}$ with $a \neq b$, we have the following double inequality:

$$
f\left(\frac{a+b}{2}\right) \leq \frac{1}{b-a} \int_{a}^{b} f(t) d t \leq \frac{f(a)+f(b)}{2} .
$$


Both inequalities hold in reverse direction if the function $f$ is concave on the interval $\mathrm{I}$. This remarkable result was given in $([3], 1893)$ and is well known in the literature as Hermite-Hadamard inequality. Since its discovery, this inequality has become the center of interest for many prolific researchers and received a considerable attention. Also, a number of extensions, generalizations, and variants of (3) have been provided in the theory of mathematical inequalities. For example, see [4-12] and the references cited therein.

Now we recall some definitions and important results in the theory of conformable fractional calculus. For detailed treatment of the results, we refer the interested readers to [1320].

Definition 3 (see [20]). Given a function $f:[0, \infty) \rightarrow \mathbb{R}$, the conformable fractional derivative of $f$ of order $\alpha$ is defined by

$$
\mathrm{D}_{\alpha}(f)(t)=\lim _{\epsilon \rightarrow 0} \frac{f\left(t+\epsilon t^{1-\alpha}\right)-f(t)}{\epsilon}
$$

for all $t>0$ and $\alpha \in(0,1)$. If the conformable fractional derivative of $f$ of order $\alpha$ exists, then we say that $f$ is $\alpha$-differentiable. Let $f$ be $\alpha$-differentiable in $(0, a)$, and $\lim _{t \rightarrow 0^{+}} f^{\alpha}(t)$ exists; then define

$$
f^{\alpha}(0)=\lim _{t \rightarrow 0^{+}} f^{\alpha}(t) .
$$

We will, sometimes, write $f^{\alpha}(t)$ and $\left(d_{\alpha} / d_{\alpha} t\right)(f)$ for $\mathrm{D}_{\alpha}(f)(t)$ to denote the conformable fractional derivatives of $f$ of order $\alpha$.

Theorem 4 (see [20]). Let $\alpha \in(0,1]$ and $f, g$ be $\alpha$ differentiable at a point $t>0$. Then we have the following:

(1) $\left(d_{\alpha} / d_{\alpha} t\right)\left(t^{n}\right)=n t^{n-\alpha}$, for all $n \in \mathbb{R}$.

(2) $\left(d_{\alpha} / d_{\alpha} t\right)(c)=0$, for all constant functions $f(t)=c$.

(3) $\left(d_{\alpha} / d_{\alpha} t\right)(a f(t)+b g(t))=a\left(d_{\alpha} / d_{\alpha} t\right)(f(t))+b\left(d_{\alpha} /\right.$ $\left.d_{\alpha} t\right)(g(t))$, for all $a, b \in \mathbb{R}$.

(4) $\left(d_{\alpha} / d_{\alpha} t\right)(f(t) g(t))=f(t)\left(d_{\alpha} / d_{\alpha} t\right)(g(t))+g(t)\left(d_{\alpha} /\right.$ $\left.d_{\alpha} t\right)(f(t))$.

(5) $\left(d_{\alpha} / d_{\alpha} t\right)(f(t) / g(t))=\left(g(t)\left(d_{\alpha} / d_{\alpha} t\right)(f(t))-f(t)\left(d_{\alpha} /\right.\right.$ $\left.\left.d_{\alpha} t\right)(g(t))\right) /(g(t))^{2}$.

(6) $\left(d_{\alpha} / d_{\alpha} t\right)((f \circ g)(t))=f^{\prime}(g(t))\left(d_{\alpha} / d_{\alpha} t\right)(g(t))$, for $f$ differentiable at $g(t)$.

If, in addition, the function $f$ is differentiable, then

$$
\frac{d_{\alpha}}{d_{\alpha} t}(f(t))=t^{1-\alpha} \frac{d}{d t}(f(t))
$$

Also, it is important to note the following:

(1) $\left(d_{\alpha} / d_{\alpha} t\right)(1)=0$.

(2) $\left(d_{\alpha} / d_{\alpha} t\right)\left(e^{a x}\right)=a x^{1-\alpha} e^{a x}, a \in \mathbb{R}$.

(3) $\left(d_{\alpha} / d_{\alpha} t\right)(\sin (a x))=a x^{1-\alpha} \cos (a x), a \in \mathbb{R}$.

(4) $\left(d_{\alpha} / d_{\alpha} t\right)(\cos (a x))=-a x^{1-\alpha} \sin (a x), a \in \mathbb{R}$.

(5) $\left(d_{\alpha} / d_{\alpha} t\right)\left((1 / \alpha) t^{\alpha}\right)=1$.
(6) $\left(d_{\alpha} / d_{\alpha} t\right)\left(\sin \left(t^{\alpha} / \alpha\right)\right)=\cos \left(t^{\alpha} / \alpha\right)$.

(7) $\left(d_{\alpha} / d_{\alpha} t\right)\left(\cos \left(t^{\alpha} / \alpha\right)\right)=-\sin \left(t^{\alpha} / \alpha\right)$.

(8) $\left(d_{\alpha} / d_{\alpha} t\right)\left(e^{\left(t^{\alpha} / \alpha\right)}\right)=e^{\left(t^{\alpha} / \alpha\right)}$.

Definition 5 (see [21] (conformable fractional integral)). Let $\alpha \in(0,1)$ and $0 \leq a<b$. A function $f:[a, b] \rightarrow \mathbb{R}$ is $\alpha$-fractional integrable on $[a, b]$ if the integral

$$
\int_{a}^{b} f(x) d_{\alpha} x:=\int_{a}^{b} f(x) x^{\alpha-1} d x
$$

exists and is finite. All $\alpha$-fractional integrable functions on $[a, b]$ are indicated by $\mathrm{L}_{\alpha}^{1}([a, b])$.

Remark 6. Note that the relation between the Riemann integral and conformable fractional integral is given by

$$
\mathrm{I}_{\alpha}^{a}(f)(t)=\mathrm{I}_{1}^{a}\left(t^{\alpha-1} f\right)=\int_{a}^{t} \frac{f(x)}{x^{1-\alpha}} d x, \quad \alpha \in(0,1] .
$$

The $\alpha$-fractional integrable functions are strongly related to fractional Lebesgue and Sobolev spaces. General definitions of fractional Lebesgue and Sobolev spaces can be found in the monograph [22]. Moreover, in recent years, they have been widely used in the theory of regularity for PDE. For interested readers, we recommend [23-25] and some of the references therein.

Theorem 7 (see [13]). Let $f:(a, b) \rightarrow \mathbb{R}$ be differentiable and $0<\alpha \leq 1$. Then, for all $t>a$, one has

$$
\mathrm{I}_{\alpha}^{a} \mathrm{D}_{\alpha}^{a}(f)(t)=f(t)-f(a) .
$$

Theorem 8 (see [13] (integration by parts)). Let $f, g$ : $[a, b] \rightarrow \mathbb{R}$ be two functions such that $f g$ is differentiable. Then

$$
\begin{aligned}
& \int_{a}^{b} f(x) \mathrm{D}_{\alpha}^{a}(g)(x) d_{\alpha} x \\
& \quad=\left.f g\right|_{a} ^{b}-\int_{a}^{b} g(x) \mathrm{D}_{\alpha}^{a}(f)(x) d_{\alpha} x
\end{aligned}
$$

Theorem 9 (see [13]). Assume that $f:[a, \infty) \rightarrow \mathbb{R}$ such that $f^{(n)}(t)$ is continuous and $\alpha \in(n, n+1]$. Then, for all $t \geq a$, one has

$$
\mathrm{D}_{\alpha}^{a} \mathrm{I}_{\alpha}^{a}(f)(t)=f(t) .
$$

Very recently, Anderson [21] investigated the following conformable integral version of Hermite-Hadamard inequality.

Theorem 10 (see [21]). Let $\alpha \in(0,1]$ and let $f:[a, b] \rightarrow \mathbb{R}$ be an $\alpha$-differentiable function with $0<a<b$, such that $\mathrm{D}_{\alpha} f$ is increasing; then one has the following inequality:

$$
\frac{\alpha}{b^{\alpha}-a^{\alpha}} \int_{a}^{b} f(t) d_{\alpha} t \leq \frac{f(a)+f(b)}{2} .
$$

Moreover, if the function $f$ is decreasing on $[a, b]$, then one has

$$
f\left(\frac{a+b}{2}\right) \leq \frac{\alpha}{b^{\alpha}-a^{\alpha}} \int_{a}^{b} f(t) d_{\alpha} t .
$$


Remark 11. It is obvious that if we choose $\alpha=1$, then inequalities (12) and (13) reduce to inequality (3).

Several important variants of Hermite-Hadamard inequality have been provided in the literature, such as the versions established by Anderson [21] and Sarikaya et al. [26] and so forth.

In this paper, we prove new Hermite-Hadamard inequalities for conformable fractional integrals by using convex function, s-convex, and coordinate convex functions. We prove new Montgomery identity for conformable fractional integral. By using this identity, we obtain Hermite-Hadamard type inequalities. These results give us the generalizations of the earlier results.

\section{Hermite-Hadamard Inequalities}

Theorem 12. Let $\alpha \in(0,1], f:[a, b] \rightarrow[0,+\infty)$ be a convex function defined on $[a, b]$, where $0<a<b$; then the following double inequality holds:

$$
\begin{aligned}
& \alpha(a+b)^{\alpha-1} f\left(\frac{a+b}{2}\right) \leq \frac{\alpha}{b-a} \int_{a}^{b} f(t) d_{\alpha} t \\
& \leq \min \left\{a^{\alpha-1}\left(\frac{\alpha f(a)+f(b)}{\alpha+1}\right),\right. \\
& \left.b^{\alpha-1}\left(\frac{f(a)+\alpha f(b)}{\alpha+1}\right)\right\} .
\end{aligned}
$$

Proof. Let us define a function $g$ on $[a, b]$ by

$$
g(t)=\left(\frac{t}{t-a}\right)^{\alpha-1} .
$$

Obviously the function $g$ is increasing and continuous function on $[a, b]$. Therefore,

$$
k_{1}:=\max _{t \in[a, b]} g(t)=\left(\frac{b}{b-a}\right)^{\alpha-1}
$$

and hence

$$
t^{\alpha-1} \leq k_{1}(t-a)^{\alpha-1}, \quad \forall t \in[a, b], \alpha \in(0,1] .
$$

Now

$$
\begin{aligned}
\int_{a}^{b} f(t) d_{\alpha} t & =\int_{a}^{b} f(t) t^{\alpha-1} d t \\
& \leq k_{1} \int_{a}^{b} f(t)(t-a)^{\alpha-1} d t
\end{aligned}
$$

(by using (17))

By changing of variable and convexity of $f$, we get

$$
\begin{aligned}
& \int_{a}^{b} f(t) d_{\alpha} t \leq k_{1}(b-a)^{\alpha} \int_{0}^{1} f(t b+(1-t) a) t^{\alpha-1} d t \\
& \quad \leq k_{1}(b-a)^{\alpha} \int_{0}^{1}(t f(b)+(1-t) f(a)) t^{\alpha-1} d t \\
& \quad=b^{\alpha-1}(b-a)\left[\frac{f(a)}{\alpha(\alpha+1)}+\frac{f(b)}{\alpha+1}\right] .
\end{aligned}
$$

Hence,

$$
\frac{\alpha}{b-a} \int_{a}^{b} f(t) d_{\alpha} t \leq b^{\alpha-1}\left(\frac{f(a)+\alpha f(b)}{\alpha+1}\right) .
$$

Now let us define a function $h$ on $[a, b]$ by

$$
h(t)=\left(\frac{t}{b-t}\right)^{\alpha-1}
$$

Clearly the function $h$ is decreasing and continuous on $[a, b]$. Therefore,

$$
k_{2}:=\max _{t \in[a, b]} h(t)=\left(\frac{a}{b-a}\right)^{\alpha-1}
$$

and hence

$$
t^{\alpha-1} \leq k_{2}(b-t)^{\alpha-1}, \quad \forall t \in[a, b], \alpha \in(0,1] .
$$

Now

$$
\begin{aligned}
& \int_{a}^{b} f(t) d_{\alpha} t=\int_{a}^{b} f(t) t^{\alpha-1} d t \\
& \quad \leq k_{2} \int_{a}^{b} f(t)(b-t)^{\alpha-1} d t \\
& \quad=k_{2}(b-a)^{\alpha} \int_{0}^{1} f(t a+(1-t) b) t^{\alpha-1} d t \\
& \quad \leq k_{2}(b-a)^{\alpha} \int_{0}^{1}(t f(a)+(1-t) f(b)) t^{\alpha-1} d t \\
& \quad=a^{\alpha-1}(b-a)\left[\frac{f(a)}{\alpha+1}+\frac{f(b)}{\alpha(\alpha+1)}\right] .
\end{aligned}
$$

Hence,

$$
\frac{\alpha}{b-a} \int_{a}^{b} f(t) d_{\alpha} t \leq a^{\alpha-1}\left(\frac{\alpha f(a)+f(b)}{\alpha+1}\right) .
$$

From (20) and (25), we deduce the right-hand side of (14). Now we prove left inequality in (14).

It is well known that

$$
\int_{a}^{b} f(t) d_{\alpha} t=\int_{a}^{(a+b) / 2} f(t) d_{\alpha} t+\int_{(a+b) / 2}^{b} f(t) d_{\alpha} t .
$$

Also from the functions $g$ and $h$ as defined in (15) and (21), respectively, we have

$$
k:=\min _{t \in[(a+b) / 2, b]} g(t)=\min _{t \in[a,(a+b) / 2]} h(t)=\left(\frac{a+b}{b-a}\right)^{\alpha-1} .
$$

Therefore,

$$
\begin{aligned}
\int_{a}^{(a+b) / 2} f(t) d_{\alpha} t & =\int_{a}^{(a+b) / 2} f(t) t^{\alpha-1} d t \\
& \geq k \int_{a}^{(a+b) / 2} f(t)(b-t)^{\alpha-1} d t, \\
\int_{(a+b) / 2}^{b} f(t) d_{\alpha} t & =\int_{(a+b) / 2}^{b} f(t) t^{\alpha-1} d t \\
& \geq k \int_{(a+b) / 2}^{b} f(t)(t-a)^{\alpha-1} d t .
\end{aligned}
$$


By using (28) in (26), we obtain

$$
\begin{gathered}
\int_{a}^{b} f(t) d_{\alpha} t \geq k\left[\int_{a}^{(a+b) / 2} f(t)(b-t)^{\alpha-1} d t\right. \\
\left.+\int_{(a+b) / 2}^{b} f(t)(t-a)^{\alpha-1} d t\right] .
\end{gathered}
$$

Now, by changing of variable and using the fact that $(2-x)^{\alpha-1} \geq 2^{\alpha-1}$ for $x \in[0,1]$, we obtain

$$
\begin{aligned}
& \int_{a}^{(a+b) / 2} f(t)(b-t)^{\alpha-1} d t=\int_{0}^{1} f\left(a+\frac{x(b-a)}{2}\right) \\
& \cdot\left(\frac{(b-a)(2-x)}{2}\right)^{\alpha-1}\left(\frac{b-a}{2}\right) d x \\
& \geq\left(\frac{b-a}{2}\right)^{\alpha} 2^{\alpha-1} \int_{0}^{1} f\left(a+\frac{t(b-a)}{2}\right) d t \\
& \quad=\frac{(b-a)^{\alpha}}{2} \int_{0}^{1} f\left(a+\frac{t(b-a)}{2}\right) d t
\end{aligned}
$$

Similarly,

$$
\begin{aligned}
& \int_{(a+b) / 2}^{b} f(t)(t-a)^{\alpha-1} d t=\int_{1}^{0} f\left(b-\frac{x(b-a)}{2}\right) \\
& \cdot\left(\frac{(b-a)(2-x)}{2}\right)^{\alpha-1}\left(\frac{-(b-a)}{2}\right) d x \\
& \quad \geq\left(\frac{b-a}{2}\right)^{\alpha} 2^{\alpha-1} \int_{0}^{1} f\left(b-\frac{t(b-a)}{2}\right) d t \\
& \quad=\frac{(b-a)^{\alpha}}{2} \int_{0}^{1} f\left(b-\frac{t(b-a)}{2}\right) d t .
\end{aligned}
$$

Now

$$
\begin{aligned}
& f\left(\frac{a+b}{2}\right)=\int_{0}^{1} f\left(\frac{a}{2}+\frac{b}{2}\right) d t \\
& \leq \frac{1}{2} \int_{0}^{1}\left[f\left(a+\frac{t(b-a)}{2}\right)+f\left(b-\frac{t(b-a)}{2}\right)\right] d t .
\end{aligned}
$$

Combining (29), (30), (31), and (32), we get

$$
\begin{aligned}
& \int_{a}^{b} f(t) d_{\alpha} t \geq k(b-a)^{\alpha}\left[\frac{1}{2}\right. \\
& \left.\cdot \int_{0}^{1}\left(f\left(a+\frac{t(b-a)}{2}\right)+f\left(b-\frac{t(b-a)}{2}\right)\right) d t\right] \\
& \quad \geq(a+b)^{\alpha-1}(b-a) f\left(\frac{a+b}{2}\right)
\end{aligned}
$$

which is equivalent to the left inequality in (14).

Corollary 13. Under the assumptions of Theorem 12, if we put $\alpha=1$, we get the following well-known Hermite-Hadamard inequality for convex function:

$$
f\left(\frac{a+b}{2}\right) \leq \frac{1}{b-a} \int_{a}^{b} f(t) d t \leq \frac{f(a)+f(b)}{2} .
$$

Now we prove Hermite-Hadamard inequality for conformable fractional integral by using $s$-convex function.

Theorem 14. Let $s, \alpha \in(0,1]$ and let $f:[a, b] \rightarrow[0,+\infty)$ be an $s$-convex function defined on $[a, b]$, where $0<a<b$; then the following double inequality holds:

$$
\begin{aligned}
& (a+b)^{\alpha-1} 2^{s-1} f\left(\frac{a+b}{2}\right) \leq \frac{1}{b-a} \int_{a}^{b} f(t) d_{\alpha} t \\
& \leq \min \left\{a^{\alpha-1}\left(\frac{f(a)}{s+\alpha}+f(b) \beta(\alpha, s+1)\right),\right. \\
& \left.b^{\alpha-1}\left(f(a) \beta(\alpha, s+1)+\frac{f(b)}{s+\alpha}\right)\right\},
\end{aligned}
$$

where $\beta(x, y)$ is Euler beta function defined for $x, y>0$.

Proof. By definition of $s$-convex function, we have

$$
\begin{aligned}
& f(t a+(1-t) b) \leq t^{s} f(a)+(1-t)^{s} f(b), \\
& f(t b+(1-t) a) \leq t^{s} f(b)+(1-t)^{s} f(a),
\end{aligned}
$$

$\forall t \in(0,1)$.

Let $g$ be defined in (15). Then, as in the proof of Theorem 12, we have

$$
\begin{aligned}
k_{1} & :=\max _{t \in[a, b]} g(t)=\left(\frac{b}{b-a}\right)^{\alpha-1}, \\
t^{\alpha-1} & \leq k_{1}(t-a)^{\alpha-1}, \quad \forall t \in[a, b], \alpha \in(0,1] .
\end{aligned}
$$

Now

$$
\begin{aligned}
\int_{a}^{b} f(t) d_{\alpha} t & =\int_{a}^{b} f(t) t^{\alpha-1} d t \\
& \leq k_{1} \int_{a}^{b} f(t)(t-a)^{\alpha-1} d t
\end{aligned}
$$

(by using (38))

By changing of variable and $s$-convexity of $f$, we get

$$
\begin{aligned}
& \int_{a}^{b} f(t) d_{\alpha} t \leq k_{1}(b-a)^{\alpha} \int_{0}^{1} f(t b+(1-t) a) t^{\alpha-1} d t \\
& \quad \leq k_{1}(b-a)^{\alpha} \int_{0}^{1}\left(t^{s} f(b)+(1-t)^{s} f(a)\right) t^{\alpha-1} d t \\
& \quad=b^{\alpha-1}(b-a)\left(f(a) \beta(\alpha, s+1)+\frac{f(b)}{s+\alpha}\right) .
\end{aligned}
$$

Hence,

$$
\begin{aligned}
& \frac{1}{b-a} \int_{a}^{b} f(t) d_{\alpha} t \\
& \quad \leq b^{\alpha-1}\left(f(a) \beta(\alpha, s+1)+\frac{f(b)}{s+\alpha}\right) .
\end{aligned}
$$


Let $h$ be defined in (21). Then similar proof leads to

$$
\begin{aligned}
k_{2} & :=\max _{t \in[a, b]} h(t)=\left(\frac{a}{b-a}\right)^{\alpha-1}, \\
t^{\alpha-1} & \leq k_{2}(b-t)^{\alpha-1}, \quad \forall t \in[a, b], \alpha \in(0,1] .
\end{aligned}
$$

Now

$$
\begin{aligned}
& \int_{a}^{b} f(t) d_{\alpha} t=\int_{a}^{b} f(t) t^{\alpha-1} d t \\
& \quad \leq k_{2} \int_{a}^{b} f(t)(b-t)^{\alpha-1} d t \\
& \quad=k_{2}(b-a)^{\alpha} \int_{0}^{1} f(t a+(1-t) b) t^{\alpha-1} d t \\
& \quad \leq k_{2}(b-a)^{\alpha} \int_{0}^{1}\left(t^{s} f(a)+(1-t)^{s} f(b)\right) t^{\alpha-1} d t \\
& \quad=a^{\alpha-1}(b-a)\left(\frac{f(a)}{s+\alpha}+f(b) \beta(\alpha, s+1)\right) .
\end{aligned}
$$

Hence,

$$
\begin{aligned}
& \frac{1}{b-a} \int_{a}^{b} f(t) d_{\alpha} t \\
& \quad \leq a^{\alpha-1}\left(\frac{f(a)}{s+\alpha}+f(b) \beta(\alpha, s+1)\right) .
\end{aligned}
$$

From (41) and (44), we deduce the right-hand side of (35). Now we prove left inequality in (35).

It is well known that

$$
\int_{a}^{b} f(t) d_{\alpha} t=\int_{a}^{(a+b) / 2} f(t) d_{\alpha} t+\int_{(a+b) / 2}^{b} f(t) d_{\alpha} t .
$$

Also from the functions $g$ and $h$ as defined in (15) and (21), respectively, we have

$$
k:=\min _{t \in[(a+b) / 2, b]} g(t)=\min _{t \in[a,(a+b) / 2]} h(t)=\left(\frac{a+b}{b-a}\right)^{\alpha-1} .
$$

Therefore,

$$
\begin{aligned}
\int_{a}^{(a+b) / 2} f(t) d_{\alpha} t & =\int_{a}^{(a+b) / 2} f(t) t^{\alpha-1} d t \\
& \geq k \int_{a}^{(a+b) / 2} f(t)(b-t)^{\alpha-1} d t \\
\int_{(a+b) / 2}^{b} f(t) d_{\alpha} t & =\int_{(a+b) / 2}^{b} f(t) t^{\alpha-1} d t \\
& \geq k \int_{(a+b) / 2}^{b} f(t)(t-a)^{\alpha-1} d t .
\end{aligned}
$$

By using (47) in (45), we obtain

$$
\begin{aligned}
& \int_{a}^{b} f(t) d_{\alpha} t \geq k\left[\int_{a}^{(a+b) / 2} f(t)(b-t)^{\alpha-1} d t\right. \\
& \left.+\int_{(a+b) / 2}^{b} f(t)(t-a)^{\alpha-1} d t\right] .
\end{aligned}
$$

Similar to (30), we have

$$
\begin{aligned}
& \int_{a}^{(a+b) / 2} f(t)(b-t)^{\alpha-1} d t \\
& \quad \geq \frac{(b-a)^{\alpha}}{2} \int_{0}^{1} f\left(a+\frac{t(b-a)}{2}\right) d t .
\end{aligned}
$$

Also,

$$
\begin{aligned}
& \int_{(a+b) / 2}^{b} f(t)(t-a)^{\alpha-1} d t \\
& \quad \geq \frac{(b-a)^{\alpha}}{2} \int_{0}^{1} f\left(b-\frac{t(b-a)}{2}\right) d t .
\end{aligned}
$$

Now, using s-convexity of $f$, we have

$$
\begin{aligned}
& f\left(\frac{a+b}{2}\right)=\int_{0}^{1} f\left(\frac{a}{2}+\frac{b}{2}\right) d t \\
& \leq \frac{1}{2^{s}} \int_{0}^{1}\left[f\left(a+\frac{t(b-a)}{2}\right)+f\left(b-\frac{t(b-a)}{2}\right)\right] d t .
\end{aligned}
$$

Combining (48), (49), (50), and (51), we get

$$
\begin{aligned}
& \int_{a}^{b} f(t) d_{\alpha} t \geq k(b-a)^{\alpha}\left[\frac{1}{2}\right. \\
& \left.\quad \cdot \int_{0}^{1}\left(f\left(a+\frac{t(b-a)}{2}\right)+f\left(b-\frac{t(b-a)}{2}\right)\right) d t\right] \\
& \quad \geq(a+b)^{\alpha-1}(b-a) 2^{s-1} f\left(\frac{a+b}{2}\right)
\end{aligned}
$$

which is equivalent to the left inequality in (35).

Corollary 15. Under the assumptions of Theorem 14, if we put $\alpha=1$, we get the following well-known Hermite-Hadamard inequality for s-convex function [27]:

$$
2^{s-1} f\left(\frac{a+b}{2}\right) \leq \frac{1}{b-a} \int_{a}^{b} f(t) d t \leq \frac{f(a)+f(b)}{s+1} .
$$

In the following theorem, we prove Hermite-Hadamard inequality for conformable fractional integral by using coordinate convex function.

Theorem 16. Let $\alpha \in(0,1]$ and let $f: \Delta=[a, b] \times[c, d] \rightarrow$ $[0,+\infty)$ be a convex function on the coordinates on $\Delta$, where 
$0<a<b$ and $0<c<d$; then the following double inequality holds:

$$
\begin{aligned}
& \frac{1}{2}\left[\frac{(c+d)^{\alpha-1}}{b-a} \int_{a}^{b} f\left(x, \frac{c+d}{2}\right) d_{\alpha} x\right. \\
& \left.\quad+\frac{(a+b)^{\alpha-1}}{d-c} \int_{c}^{d} f\left(\frac{a+b}{2}, y\right) d_{\alpha} y\right] \\
& \leq \frac{1}{(b-a)(d-c)} \int_{a}^{b} \int_{c}^{d} f(x, y) d_{\alpha} x d_{\alpha} y \\
& \leq \frac{1}{2 \alpha(\alpha+1)}\left[\frac{d^{\alpha-1}}{b-a} \int_{a}^{b}(f(x, c)+\alpha f(x, d)) d_{\alpha} x\right. \\
& \left.\quad+\frac{b^{\alpha-1}}{d-c} \int_{c}^{d}(f(a, y)+\alpha f(b, y)) d_{\alpha} y\right] .
\end{aligned}
$$

Proof. Since $f: \Delta \rightarrow[0,+\infty)$ is convex on the coordinates, it follows that the mapping $f_{x}:[c, d] \rightarrow[0,+\infty), f_{x}(y)=$ $f(x, y)$, is convex on $[c, d]$ for all $x \in[a, b]$. Then, by Theorem 12, we have

$$
(c+d)^{\alpha-1} f_{x}\left(\frac{c+d}{2}\right) \leq \frac{1}{d-c} \int_{c}^{d} f_{x}(y) d_{\alpha} y
$$

$$
x \in[a, b]
$$

that is,

$$
\begin{array}{r}
(c+d)^{\alpha-1} f\left(x, \frac{c+d}{2}\right) \leq \frac{1}{d-c} \int_{c}^{d} f(x, y) d_{\alpha} y \\
x \in[a, b] .
\end{array}
$$

Integrating (56) on $[a, b]$, we have

$$
\begin{aligned}
& \frac{(c+d)^{\alpha-1}}{b-a} \int_{a}^{b} f\left(x, \frac{c+d}{2}\right) d_{\alpha} x \\
& \leq \frac{1}{(b-a)(d-c)} \int_{a}^{b} \int_{c}^{d} f(x, y) d_{\alpha} x d_{\alpha} y .
\end{aligned}
$$

Similarly, using the mapping $f_{y}:[a, b] \rightarrow[0,+\infty), f_{y}(x)=$ $f(x, y)$, we get

$$
\begin{aligned}
& \frac{(a+b)^{\alpha-1}}{d-c} \int_{c}^{d} f\left(\frac{a+b}{2}, y\right) d_{\alpha} y \\
& \leq \frac{1}{(b-a)(d-c)} \int_{a}^{b} \int_{c}^{d} f(x, y) d_{\alpha} x d_{\alpha} y .
\end{aligned}
$$

Summing inequalities (57) and (58), we deduce the lefthand side of (54).

Now we prove right inequality in (54).
Also, by Theorem 12, using inequality (20), we have

$$
\begin{aligned}
& \frac{1}{(b-a)(d-c)} \int_{a}^{b} \int_{c}^{d} f(x, y) d_{\alpha} x d_{\alpha} y \\
& =\frac{1}{(b-a)(d-c)} \int_{c}^{d}\left[\int_{a}^{b} f_{y}(x) d_{\alpha} x\right] d_{\alpha} y \\
& \leq \frac{1}{(b-a)(d-c)} \\
& \cdot \int_{c}^{d}\left[b^{\alpha-1}(b-a)\left(\frac{f(a, y)}{\alpha(\alpha+1)}+\frac{f(b, y)}{\alpha+1}\right)\right] d_{\alpha} y \\
& =\frac{b^{\alpha-1}}{\alpha(\alpha+1)(d-c)} \int_{c}^{d}(f(a, y)+\alpha f(b, y)) d_{\alpha} y .
\end{aligned}
$$

Similarly, using inequality (25), we have

$$
\begin{aligned}
& \frac{1}{(b-a)(d-c)} \int_{a}^{b} \int_{c}^{d} f(x, y) d_{\alpha} x d_{\alpha} y \\
& =\frac{1}{(b-a)(d-c)} \int_{a}^{b}\left[\int_{c}^{d} f_{x}(y) d_{\alpha} y\right] d_{\alpha} x \\
& \leq \frac{1}{(b-a)(d-c)} \\
& \cdot \int_{a}^{b}\left[d^{\alpha-1}(d-c)\left(\frac{f(x, c)}{\alpha(\alpha+1)}+\frac{f(x, d)}{\alpha+1}\right)\right] d_{\alpha} x \\
& =\frac{d^{\alpha-1}}{\alpha(\alpha+1)(b-a)} \int_{a}^{b}(f(x, c)+\alpha f(x, d)) d_{\alpha} x .
\end{aligned}
$$

Summing inequalities (59) and (60), we deduce the righthand side of (54).

Remark 17. Under the assumptions of Theorem 16, if we put $\alpha=1$, we get the Hermite-Hadamard inequality for coordinate convex function obtained by Dragomir in [2].

Remark 18. Under the assumptions of Theorem 16, using inequalities (57), (58), (59), and (60), the following double inequality holds:

$$
\begin{aligned}
& \max \left\{\frac{(c+d)^{\alpha-1}}{b-a} \int_{a}^{b} f\left(x, \frac{c+d}{2}\right) d_{\alpha} x, \frac{(a+b)^{\alpha-1}}{d-c}\right. \\
& \left.\cdot \int_{c}^{d} f\left(\frac{a+b}{2}, y\right) d_{\alpha} y\right\} \leq \frac{1}{(b-a)(d-c)} \\
& \cdot \int_{a}^{b} \int_{c}^{d} f(x, y) d_{\alpha} x d_{\alpha} y \leq \frac{1}{\alpha(\alpha+1)} \cdot \min \left\{\frac{d^{\alpha-1}}{b-a}\right. \\
& \cdot \int_{a}^{b}(f(x, c)+\alpha f(x, d)) d_{\alpha} x, \frac{b^{\alpha-1}}{d-c} \\
& \left.\cdot \int_{c}^{d}(f(a, y)+\alpha f(b, y)) d_{\alpha} y\right\} .
\end{aligned}
$$




\section{Generalization of Hermite-Hadamard Type Inequalities}

Now, we are in position to find some new estimations for the left-hand side of Hermite-Hadamard inequality for conformable fractional integral as given in Theorem 12 by using a new Montgomery identity.

Lemma 19 (new Montgomery identity). Let $a, b \in \mathbb{R}$ with $0 \leq a<b$, and let $f:[a, b] \rightarrow \mathbb{R}$ be $\alpha$-fractional differentiable function, where $\alpha \in(0,1]$. Then

$$
\begin{aligned}
f(t)= & \frac{1}{b-a} \int_{a}^{b} f(s) d_{\alpha} s \\
& +\frac{1}{b-a} \int_{a}^{b} p(t, s) D_{\alpha} f(s) d_{\alpha} s \\
& +\frac{f(a)+f(b)}{2}+\frac{a^{\alpha} f(a)-b^{\alpha} f(b)}{\alpha(b-a)},
\end{aligned}
$$

where

$$
p(t, s):= \begin{cases}\frac{s^{\alpha}}{\alpha}+\frac{b-a}{2}: & a \leq s<t, \\ \frac{s^{\alpha}}{\alpha}-\frac{b-a}{2}: & t \leq s \leq b .\end{cases}
$$

Proof. Integrating by parts, we have

$$
\begin{aligned}
& \int_{a}^{t}\left(\frac{s^{\alpha}}{\alpha}+\frac{b-a}{2}\right) D_{\alpha} f(s) d_{\alpha} s \\
& =\left(\frac{t^{\alpha}}{\alpha}+\frac{b-a}{2}\right) f(t)-\left(\frac{a^{\alpha}}{\alpha}+\frac{b-a}{2}\right) f(a) \\
& \quad-\int_{a}^{t} f(s) d_{\alpha} s, \\
& \int_{t}^{b}\left(\frac{s^{\alpha}}{\alpha}-\frac{b-a}{2}\right) D_{\alpha} f(s) d_{\alpha} s \\
& =\left(\frac{b^{\alpha}}{\alpha}-\frac{b-a}{2}\right) f(b)-\left(\frac{t^{\alpha}}{\alpha}-\frac{b-a}{2}\right) f(t) \\
& \quad-\int_{t}^{b} f(s) d_{\alpha} s .
\end{aligned}
$$

Adding and solving for $f$ yields the required result.

Theorem 20. Let $f:[a, b] \rightarrow[0,+\infty)$ be an $\alpha$-fractional differentiable function such that $|f|^{q}$ is a convex function, where $0<a<b$ and $\alpha \in(0,1]$. Then, for $q>1$ and $p^{-1}+q^{-1}=1$, one has

$$
\begin{aligned}
& \left|(a+b)^{\alpha-1} f\left(\frac{a+b}{2}\right)-\frac{1}{b-a} \int_{a}^{b} f(t) d_{\alpha} t\right| \\
& \quad \leq C(f ; \alpha, a, b, p, q)+|K|+M \xi\left[2 \alpha(b-a) t^{\alpha}\right. \\
& \left.\quad+\left(a^{\alpha}+b^{\alpha}\right)\left(b^{\alpha}-a^{\alpha}-\alpha(b-a)\right)\right],
\end{aligned}
$$

where

$$
\begin{aligned}
M & :=\sup _{t \in[a, b]}\left|D_{\alpha} f(t)\right|, \\
\xi:= & \frac{(a+b)^{\alpha-1}}{2 \alpha^{2}(b-a)}, \\
K:= & \frac{f(a)+f(b)}{2}+\frac{a^{\alpha} f(a)-b^{\alpha} f(b)}{\alpha(b-a)}, \\
C(f ; \alpha, a, b, p, q) & : \frac{\left|(a+b)^{\alpha-1}-1\right|}{b-a}\left(\frac{b^{p(\alpha-1)+1}-a^{p(\alpha-1)+1}}{p(\alpha-1)+1}\right)^{1 / p} \\
& \quad \times\left[(b-a)\left(\frac{f^{q}(a)+f^{q}(b)}{2}\right)\right]^{1 / q} .
\end{aligned}
$$

Proof. Using Lemma 19 with $p(t, s)$ defined in (63), convexity of $f^{q}$, Hölder's inequality, and property of the modulus, we have

$$
\begin{aligned}
& \left|(a+b)^{\alpha-1} f\left(\frac{a+b}{2}\right)-\frac{1}{b-a} \int_{a}^{b} f(t) d_{\alpha} t\right| \\
& \leq \frac{\left|(a+b)^{\alpha-1}-1\right|}{b-a} \int_{a}^{b}|f(s)| d_{\alpha} s+\frac{(a+b)^{\alpha-1}}{b-a} \\
& \cdot \int_{a}^{b}\left|p\left(\frac{a+b}{2}, s\right)\right|\left|D_{\alpha} f(s)\right| d_{\alpha} s \\
& +\left|\frac{f(a)+f(b)}{2}+\frac{a^{\alpha} f(a)-b^{\alpha} f(b)}{\alpha(b-a)}\right| \\
& \leq \frac{\left|(a+b)^{\alpha-1}-1\right|}{b-a}\left(\int_{a}^{b} s^{p(\alpha-1)} d s\right)^{1 / p} \\
& \cdot\left(\int_{a}^{b} f^{q}(s) d s\right)^{1 / q}+|K|+M \\
& . \frac{(a+b)^{\alpha-1}}{b-a}\left[\int_{a}^{t}\left|\frac{s^{\alpha}}{\alpha}+\frac{b-a}{2}\right| d_{\alpha} s\right. \\
& \left.+\int_{t}^{b}\left|\frac{s^{\alpha}}{\alpha}-\frac{b-a}{2}\right| d_{\alpha} s\right] \\
& \quad+\frac{(a+b)^{\alpha-1}-1 \mid}{b-a}\left(\int_{a}^{b} s^{p(\alpha-1)} d s\right)^{1 / p} \\
& +\left(\int_{0}^{b}\left(\frac{s^{\alpha}}{\alpha}-\frac{b-a}{2}\right) d_{\alpha} s\right]=C(f ; \alpha, a, b, p, q) \\
& +|K|+M \frac{(a+b)^{\alpha-1}}{b-a}\left[\int_{a}^{t}\left(\frac{s^{\alpha}}{\alpha}+\frac{b-a}{2}\right) d_{\alpha} s\right. \\
& \left.\left.+(1-t) f^{q}(a)\right](b-a) d t\right)^{1 / q}
\end{aligned}
$$




$$
\begin{aligned}
& +|K|+M \xi\left[2 \alpha(b-a) t^{\alpha}\right. \\
& \left.+\left(a^{\alpha}+b^{\alpha}\right)\left(b^{\alpha}-a^{\alpha}-\alpha(b-a)\right)\right] .
\end{aligned}
$$

Theorem 21. Let $f:[a, b] \rightarrow[0,+\infty)$ be $\alpha$-fractional differentiable and $f^{q}$ is a convex function, where $0<a<b$ and $\alpha \in(0,1]$. Then, for $q \geq 1$, one has

$$
\begin{aligned}
\mid(a & +b)^{\alpha-1} f\left(\frac{a+b}{2}\right)-\frac{1}{b-a} \int_{a}^{b} f(t) d_{\alpha} t \mid \\
& \leq D(f ; \alpha, a, b, q)+|K|+M \xi\left[2 \alpha(b-a) t^{\alpha}\right. \\
& \left.+\left(a^{\alpha}+b^{\alpha}\right)\left(b^{\alpha}-a^{\alpha}-\alpha(b-a)\right)\right]
\end{aligned}
$$

where

$$
\begin{aligned}
& D(f ; \alpha, a, b, q) \\
&:= \frac{\left|(a+b)^{\alpha-1}-1\right|}{b-a}\left(\frac{b^{\alpha}-a^{\alpha}}{\alpha}\right)^{1-1 / q} \\
& \times\left[\frac{f^{q}(a) B_{1}(\alpha)+f^{q}(b) B_{2}(\alpha)}{b-a}\right]^{1 / q}, \\
& B_{1}(\alpha)=\int_{a}^{b} t^{\alpha-1}(b-t) d t \\
&= \frac{b\left(b^{\alpha}-a^{\alpha}\right)}{\alpha}-\frac{b^{\alpha+1}-a^{\alpha+1}}{\alpha+1}, \\
& B_{2}(\alpha)=\int_{a}^{b} t^{\alpha-1}(t-a) d t \\
&=\frac{b^{\alpha+1}-a^{\alpha+1}}{\alpha+1}-\frac{a\left(b^{\alpha}-a^{\alpha}\right)}{\alpha},
\end{aligned}
$$

and $M, \xi, K$ are defined as in Theorem 20.

Proof. Using Lemma 19 with $p(t, s)$ defined in (63), convexity of $f^{q}$, the well-known power mean inequality, and property of the modulus, we have

$$
\begin{aligned}
& \left|(a+b)^{\alpha-1} f\left(\frac{a+b}{2}\right)-\frac{1}{b-a} \int_{a}^{b} f(t) d_{\alpha} t\right| \\
& \quad \leq \frac{\left|(a+b)^{\alpha-1}-1\right|}{b-a} \int_{a}^{b}|f(s)| d_{\alpha} s+\frac{(a+b)^{\alpha-1}}{b-a} \\
& \cdot \int_{a}^{b}\left|p\left(\frac{a+b}{2}, s\right)\right|\left|D_{\alpha} f(s)\right| d_{\alpha} s \\
& +\left|\frac{f(a)+f(b)}{2}+\frac{a^{\alpha} f(a)-b^{\alpha} f(b)}{\alpha(b-a)}\right| \\
& \quad \leq \frac{\left|(a+b)^{\alpha-1}-1\right|}{b-a}\left(\int_{a}^{b} s^{\alpha-1} d s\right)^{1-1 / q} \\
& \cdot\left(\int_{a}^{b} s^{\alpha-1} f^{q}(s) d s\right)^{1 / q}+|K|+M
\end{aligned}
$$

$$
\begin{aligned}
& \cdot \frac{(a+b)^{\alpha-1}}{b-a}\left[\int_{a}^{t}\left|\frac{s^{\alpha}}{\alpha}+\frac{b-a}{2}\right| d_{\alpha} s\right. \\
& \left.+\int_{t}^{b}\left|\frac{s^{\alpha}}{\alpha}-\frac{b-a}{2}\right| d_{\alpha} s\right] \\
& \leq \frac{\left|(a+b)^{\alpha-1}-1\right|}{b-a}\left(\int_{a}^{b} s^{\alpha-1} d s\right)^{1-1 / q} \\
& \times\left(\int_{0}^{1}(t b+(1-t) a)^{\alpha-1}\left[t f^{q}(b)+(1-t) f^{q}(a)\right]\right. \\
& \cdot(b-a) d t)^{1 / q}+|K|+M \\
& \cdot \frac{(a+b)^{\alpha-1}}{b-a}\left[\int_{a}^{t}\left(\frac{s^{\alpha}}{\alpha}+\frac{b-a}{2}\right) d d_{\alpha}\right. \\
& \left.+\int_{t}^{b}\left(\frac{s^{\alpha}}{\alpha}-\frac{b-a}{2}\right) d_{\alpha} s\right]=D(f ; \alpha, a, b, q)+|K| \\
& +M \xi\left[2 \alpha(b-a) t^{\alpha}+\left(a^{\alpha}+b^{\alpha}\right)\left(b^{\alpha}-a^{\alpha}\right.\right. \\
& -\alpha(b-a))] \cdot
\end{aligned}
$$

\section{Conflicts of Interest}

The authors declare that there are no conflicts of interest regarding the publication of this paper.

\section{Acknowledgments}

The research was supported by the National Natural Science Foundation of China (Grants nos. 61673169, 61374086, 11371125, 11401191, and 11701176), the Tianyuan Special Funds of the National Natural Science Foundation of China (Grant no. 11626101), and the Natural Science Foundation of the Department of Education of Zhejiang Province (Grant no. Y201635325).

\section{References}

[1] H. Hudzik and L. Maligranda, "Some remarks on s-convex functions," Aequationes Mathematicae, vol. 48, no. 1, pp. 100111, 1994.

[2] S. S. Dragomir, "On the Hadamard's inequality for convex functions on the co-ordinates in a rectangle from the plane," Taiwanese Journal of Mathematics, vol. 5, no. 4, pp. 775-788, 2001.

[3] J. Hadamard, "Etude sur les propriétés des fonctions entières et en particulier d'une fonction considérée par Riemann," Journal de Mathématiques Pures et Appliquées, vol. 58, pp. 171-215, 1893.

[4] M. A. Khan, Y. Khurshid, and T. Ali, "Hermite-Hadamard inequality for fractional integrals via $\eta$-convex functions," Acta Mathematica Universitatis Comenianae, vol. 86, no. 1, pp. 153164, 2017. 
[5] M. A. Khan, T. Ali, S. S. Dragomir, and M. Z. Sarikaya, "Hermite-Hadamard type inequalities for conformable fractional integrals," Revista de la Real Academia de Ciencias Exactas, Físicas y Naturales. Serie A. Matemáticas, pp. 1-16, 2017.

[6] M. A. Khan, Y. Khurshid, T. Ali, and N. Rehman, "Inequalities for three times differentiable functions," The Punjab University. Journal of Mathematics, vol. 48, no. 2, pp. 35-48, 2016.

[7] Y.-M. Chu, M. A. Khan, T. Ali, and S. S. Dragomir, "Inequalities for $\alpha$-fractional differentiable functions," Journal of Inequalities and Applications, vol. 2017, Article ID 93, 12 pages, 2017.

[8] S. S. Dragomir, "Two mappings in connection to Hadamard's inequalities," Journal of Mathematical Analysis and Applications, vol. 167, no. 1, pp. 49-56, 1992.

[9] S. S. Dragomir and R. P. Agarwal, "Two inequalities for differentiable mappings and applications to special means of real numbers and to trapezoidal formula," Applied Mathematics Letters, vol. 11, no. 5, pp. 91-95, 1998.

[10] S. S. Dragomir and C. E. M. Pearce, "Selected topics on HermiteHadamard inequalities and applications," RGMIA Monographs, 2000, http://rgmia.org/monographs/hemite_hadamard.html.

[11] S. S. Dragomir, J. Pecaric, and L. E. Persson, "Some inequalities of Hadamard type," Soochow Journal of Mathematics, vol. 21, no. 3, pp. 335-341, 1995.

[12] M. Z. Sarikaya, A. Saglam, and H. Yildirim, "On some Hadamard-type inequalities for h-convex functions," Journal of Mathematical Inequalities, vol. 2, no. 3, pp. 335-341, 2008.

[13] T. Abdeljawad, "On conformable fractional calculus," Journal of Computational and Applied Mathematics, vol. 279, pp. 57-66, 2015.

[14] T. Abdeljawad, M. Al Horani, and R. Khalil, "Conformable fractional semigroups of operators," Journal of Semigroup Theory and Applications, vol. 2015, 9 pages, 2015.

[15] D. R. Anderson and D. J. Ulness, "Newly defined conformable derivatives," Advances in Dynamical Systems and Applications (ADSA), vol. 10, no. 2, pp. 109-137, 2015.

[16] D. R. Anderson, "Second-order self-adjoint differential equations using a proportional-derivative controller," Communications on Applied Nonlinear Analysis, vol. 24, no. 1, pp. 17-48, 2017.

[17] M. A. Hammad and R. Khalil, "Conformable fractional heat differential equation," International Journal of Pure and Applied Mathematics, vol. 94, no. 2, pp. 215-221, 2014.

[18] M. A. Hammad and R. Khalil, "Abel's formula and Wronskian for conformable fractional differential equations," International Journal of Differential Equations and Applications, vol. 13, no. 3, pp. 177-183, 2014.

[19] O. S. Iyiola and E. R. Nwaeze, "Some new results on the new conformable fractional calculus with application using d'alambert approach," Progress in Fractional Differentiation and Applications, vol. 2, no. 2, pp. 115-122, 2016.

[20] R. Khalil, M. Al Horani, A. Yousef, and M. Sababheh, "A new definition of fractional derivative," Journal of Computational and Applied Mathematics, vol. 264, pp. 65-70, 2014.

[21] D. R. Anderson, "Taylor's formula and integral inequalities for conformable fractional derivatives," in Contributions in Mathematics and Engineering, pp. 25-43, Springer, Cham, Switzerland, 2016.

[22] R. A. Adams, Sobolev Spaces, Academic Press, New York, NY, USA, 1975.

[23] S. Leonardi and J. Stara, "Regularity results for the gradient of solutions of linear elliptic systems with VMO coefficients and $\mathrm{L}(1, \lambda)$ data," Forum Mathematicum, vol. 22, no. 5, pp. 913-940, 2010.
[24] S. Leonardi and J. Stara, "Regularity up to the boundary for the gradient of solutions of linear elliptic systems with VMO coefficients and $\mathrm{L}(1, \lambda)$ data," Complex Variables and Elliptic Equations, vol. 56, no. 12, pp. 1085-1098, 2011.

[25] S. Leonardi and J. Stara, "Regularity results for solutions of a class of parabolic systems with measure data," Nonlinear Analysis, vol. 75, no. 4, pp. 2069-2089, 2012.

[26] M. Z. Sarikaya, E. Set, H. Yaldiz, and N. Basak, "HermiteHadamard's inequalities for fractional integrals and related fractional inequalities," Mathematical and Computer Modelling, vol. 57, no. 9-10, pp. 2403-2407, 2013.

[27] S. S. Dragomir and S. Fitzpatrick, "The Hadamard inequalities for s-convex functions in the second sense," Demonstratio Mathematica, vol. 32, no. 4, pp. 687-696, 1999. 


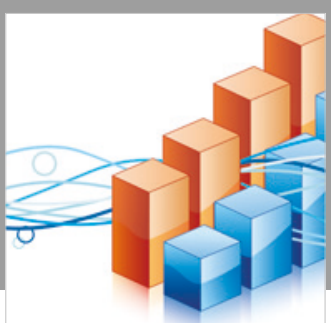

Advances in

Operations Research

\section{-n-m}
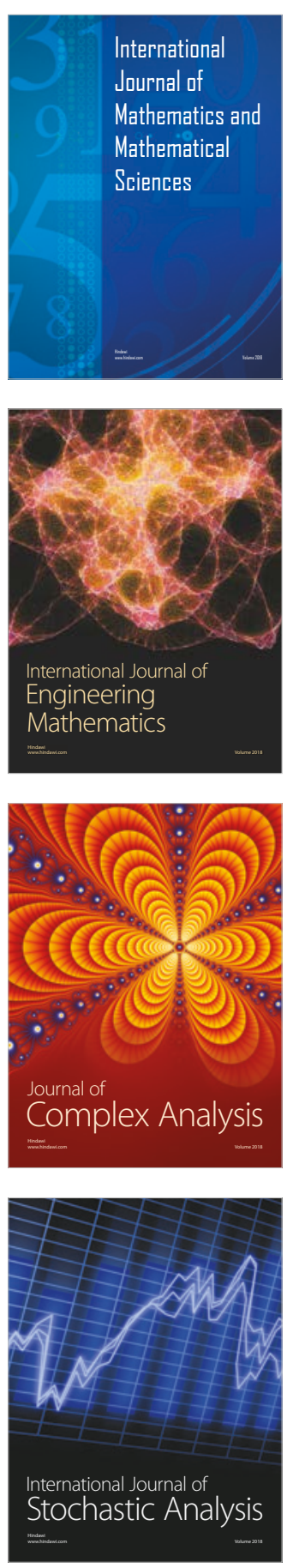
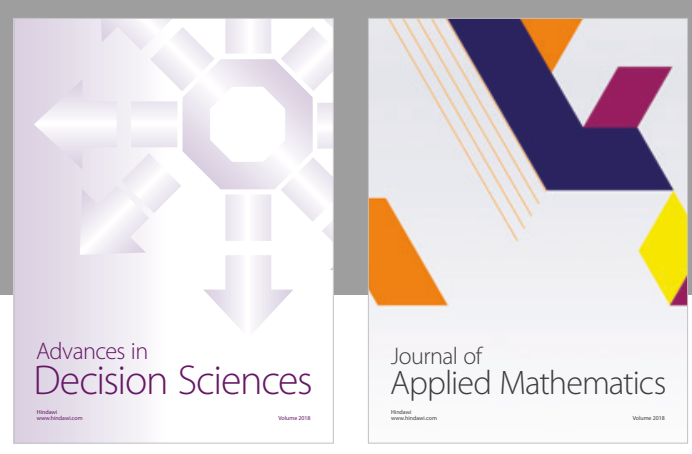

Journal of

Applied Mathematics
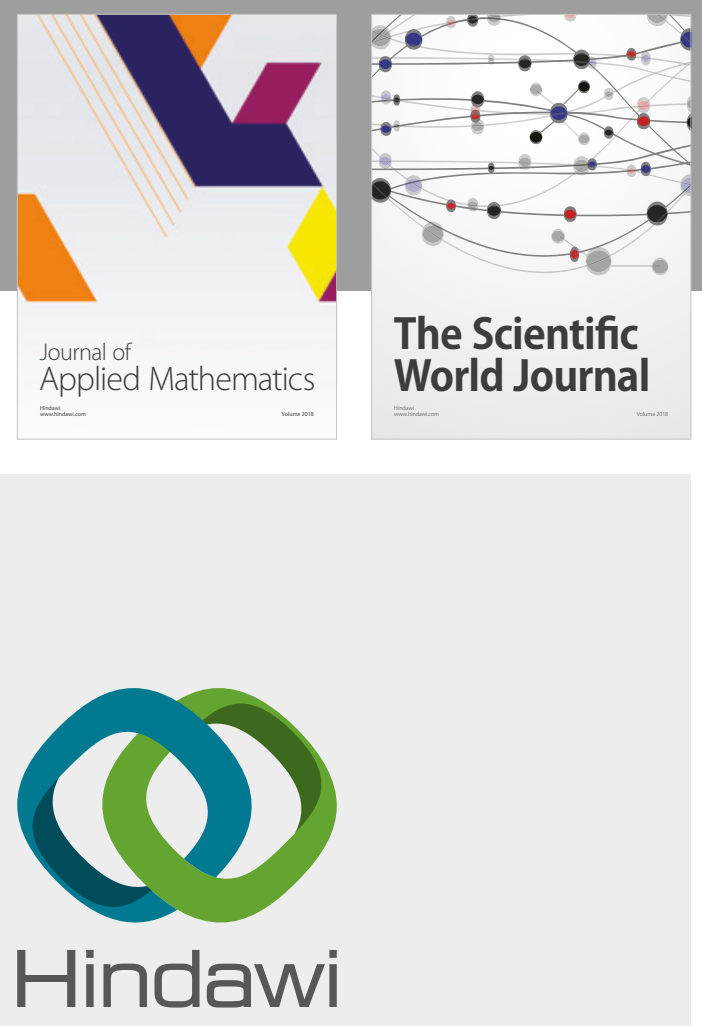

Submit your manuscripts at

www.hindawi.com

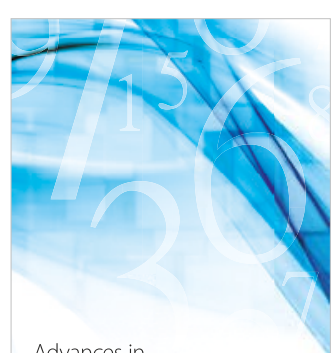

Advances in
Numerical Analysis
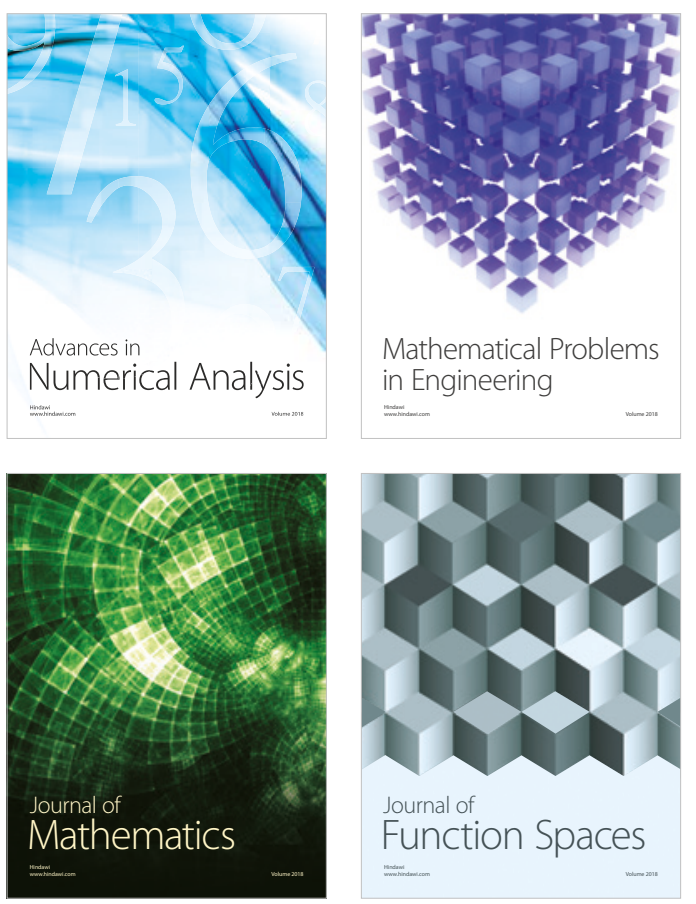

Mathematical Problems in Engineering

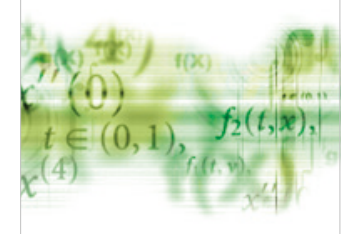

International Journal of

Differential Equations

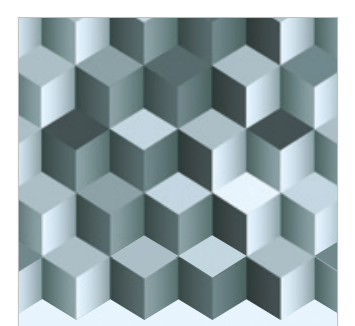

Journal of

Function Spaces

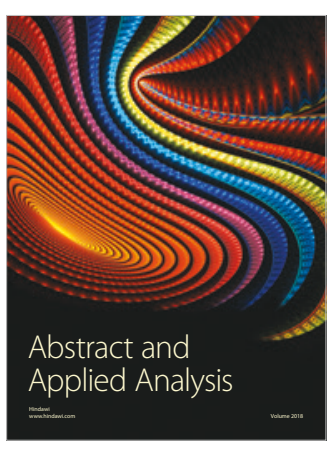

The Scientific

World Journal

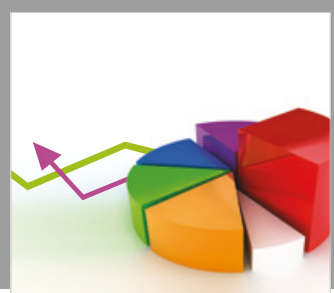

Journal of

Probability and Statistics
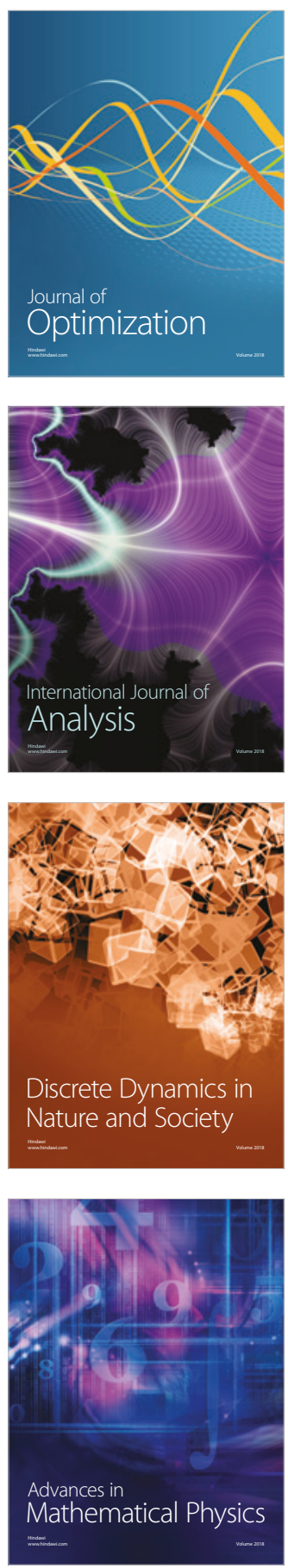